\title{
Quantum phase transition in a 1D transport model with boson affected hopping: Luttinger liquid versus charge-density-wave behavior
}

\author{
S. Ejima, ${ }^{1}$ G. Hager, ${ }^{2}$ and H. Fehske ${ }^{1}$ \\ 1 Institut für Physik, Ernst-Moritz-Arndt-Universität Greifswald, 17489 Greifswald, Germany \\ 2 Regionales Rechenzentrum Erlangen, Universität Erlangen-Nürnberg, 91058 Erlangen, Germany
}

\begin{abstract}
We solve a very general two-channel fermion-boson model describing charge transport within some background medium by means of a refined pseudo-site density matrix renormalization group (DMRG) technique. Performing a careful finite-size scaling analysis, we determine the ground-state phase diagram and convincingly prove that the model exhibits a metal-insulator quantum phase transition for the half-filled band case. In order to characterize the metallic and insulating regimes we calculate besides the local particle densities and fermion-boson correlation functions, the kinetic energy, the charge structure factor, the Luttinger liquid charge exponent and the single-particle excitation gap for a one-dimensional infinite system.
\end{abstract}

PACS numbers: 71.10.-w,71.30.+h,71.10.Fd,71.10.Hf

The proof of existence of metal-insulator transitions (MITs) in generic model Hamiltonians is one of the most fundamental problems in solid state theory. While the mechanisms that can drive a MIT, such as band structure effects [1], disorder [2], Coulomb correlations 3], or the coupling to the lattice degrees of freedom [4], are accepted in general, there is only a very small number of microscopic models which have rigorously been shown to indeed exhibit such a transition. Examples are the 3D Anderson tight-binding (disorder) model for which an analytical proof of particle localization exists [5], or the 1D spinless fermion Holstein (electronphonon) model, where the Tomonaga-Luttinger-liquid (TLL) charge-density-wave (CDW) MIT has been confirmed numerically by DMRG 6]. Zero-temperature MITs triggered by Coulomb interaction are more difficult to assess; the prototype half-filled 1D Hubbard model, e.g., is insulating for all $U>0$ [7], and only on introducing a (particular) long-range hopping the MIT takes place at finite interaction strength [8]. In the 1D halffilled extended Hubbard $(U-V)$ model, there exists at most a metallic line at the bond-order- charge-densitywave insulator-insulator transition [9]. If Coulomb and electron-phonon interactions compete, an extended intervening metallic phase may occur between Mott and Peierls insulating states, which allows for a MIT. This has been demonstrated for the 1D Holstein-Hubbard model at half filling [10].

Quite recently a novel quantum transport Hamiltonian has been proposed [11], which describes regimes of quasifree, correlation or fluctuation dominated transport. In a sense this model parameterizes the correlations inherent to a fermionic many-particle system, but also the couplings to phonon or bath degrees of freedom, by a "background medium" that controls the particle's transport properties. Thus the model captures basic aspects of more complicated Hubbard or Holstein Hamiltonians. Then it is a legitimate question to ask whether the interaction with the background may even drive a MIT.
Consider the Hamiltonian 11

$$
H=H_{b}-\lambda \sum_{i}\left(b_{i}^{\dagger}+b_{i}\right)+\omega_{0} \sum_{i} b_{i}^{\dagger} b_{i}+\frac{N \lambda^{2}}{\omega_{0}},
$$

where $H_{b}=-t_{b} \sum_{\langle i, j\rangle} f_{j}^{\dagger} f_{i}\left(b_{i}^{\dagger}+b_{j}\right)$ describes a bosonaffected nearest-neighbor hopping $\left(\propto t_{b}\right)$ of spinless fermionic particles $\left(f_{i}^{(\dagger)}\right)$. A fermion emits (or absorbs) a local boson $b_{j}^{\dagger}\left(b_{i}\right)$ every time it hops between lattice sites $i, j$. This way the particle creates local distortions of certain energy in the background. In the case of an antiferromagnetic spin background the distortions correspond to local spin deviations (cf. the motion of a hole in the $t$ - $J$ model [12]). If the background medium is a deformable lattice they are basically lattice fluctuations (phonons). Other situations such as doped CDWs or exciton transport in molecular aggregates might be envisaged. In any case the distortions of the background can be parameterized as bosons [11, 13]. The distortions are able to relax (compare $\lambda$ with $J_{\perp}$ in the $t$ - $J$ model), which is described by the second term in (11). The third term gives the energy of the bosons; the constant energy shift $N \lambda^{2} / \omega_{0}$ guarantees finite energy for $N \rightarrow \infty$. Performing the unitary transformation $b_{i} \mapsto b_{i}+\lambda / \omega_{0}$ eliminates the boson relaxation term in favor of a second, free-fermion hopping channel, $H \rightarrow H=H_{b}+H_{f}+\omega_{0} \sum_{i} b_{i}^{\dagger} b_{i}$, where $H_{f}=-t_{f} \sum_{\langle i, j\rangle} f_{j}^{\dagger} f_{i}$ with $t_{f}=2 \lambda t_{b} / \omega_{0}$. Hereafter we focus on the 1D half-filled band case, i.e., fermion number $N_{f} / N=1 / 2$, and take $t_{b}=1$ as energy unit.

In our model (1), the particles have only a charge degree of freedom. Then, for a tight-binding band structure and in the absence of disorder, the formation of a CDW is the only possibility for a MIT. The CDW might be induced by strong correlations in the background, which exist for large $\omega_{0}$ because (i) coherent transport $\left(\propto t_{f}\right)$ takes place on a strongly reduced energy scale only and (ii) incoherent transport is energetically costly [11]. Hence, in the limit $\omega_{0} \gg 1$, an effective Hamiltonian with nearest-neighbor fermion repulsion results. By contrast, 
if the local distortions of the background relax readily (i.e., $\lambda \gg 1$ ) and/or the energy of the bosons is small (i.e., $\omega_{0} \ll 1$ ), the free hopping channel can act efficiently against any correlation-induced charge ordering.

Evidence for a MIT comes from a very recent exact diagonalization (ED) study of (1): Calculating the wavevector-resolved photoemission and inverse photoemission spectra the opening of a single-particle excitation gap has been observed at $K_{F}= \pm \pi / 2$ as $\lambda$ decreases at relatively large $\omega_{0}=2$ [14]. Of course, dealing with lattices up to 16 sites, this does not unambiguously prove the existence of a true phase transition which may occur in the thermodynamic limit $N \rightarrow \infty$ only.

In this work, we carry out the first large-scale DMRG investigation of the two-channel transport model (1). In combination with a finite-size scaling analysis this allows us to map out the ground-state phase diagram for the 1D half-filled band case and to characterize the different phases involved. The DMRG is one of the most powerful and accurate numerical techniques for studying 1D fermionic many-body systems [15]. It can be easily generalized to treat systems including bosons. Within the pseudo-site approach an exact mapping of a boson site, containing $2^{n_{b}}$ states, to $n_{b}$ pseudo-sites is performed [16]. Here we take into account up to $n_{b}=4$ pseudo-sites, so that the $n_{b}$-th local boson density is always smaller than $10^{-8}$. In addition, we keep $m=1200$ to 2000 density-matrix eigenstates and extrapolate all quantities to the $m \rightarrow \infty$ limit. To test our DMRG implementation we compared data obtained for small systems with previous ED results [14] and got very good agreement: The relative error of the ground-state energy $\left|E_{\mathrm{ED}}-E_{\mathrm{DMRG}}\right|$ was always smaller than $10^{-7}$ (for all $\lambda$ at $\omega_{0}=2$ ); the discarded weight was smaller than $5 \times 10^{-8}$.

As indicated by small cluster EDs [14], at $\omega_{0}=2.0$, where fermions and bosons are strongly correlated for small $\lambda$, a MIT might occur in the range of $0.01<\lambda<5$. This is confirmed by DMRG for much larger systems: Figs. 1 (a) and (b), showing the variation of the local densities of fermions $\left\langle f_{i}^{\dagger} f_{i}\right\rangle$ and bosons $\left\langle b_{i}^{\dagger} b_{i}\right\rangle$, respectively, point towards the existence of a homogeneous state (CDW state) for rather large (small) $\lambda$. Using open boundary conditions (OBC), the system is obviously not translation invariant, i.e., the local density is inhomogeneous in any case. In the CDW phase, there are two degenerate ground states. Within an OBC DMRG calculation, one of these ground states is picked out by initializing the DMRG algorithm, so that the CDW state is directly observable in the local density. In the metallic regime, on the other hand, the open boundaries reveal (strong) Friedel oscillations, which will be algebraically reduced, however, as we move towards the interior. Thus for large enough system sizes, within the central part of the chain, the local density becomes constant (see filled symbols in Figs. 1 (a) and (b)) 17].

The CDW structure of the insulating state shows up
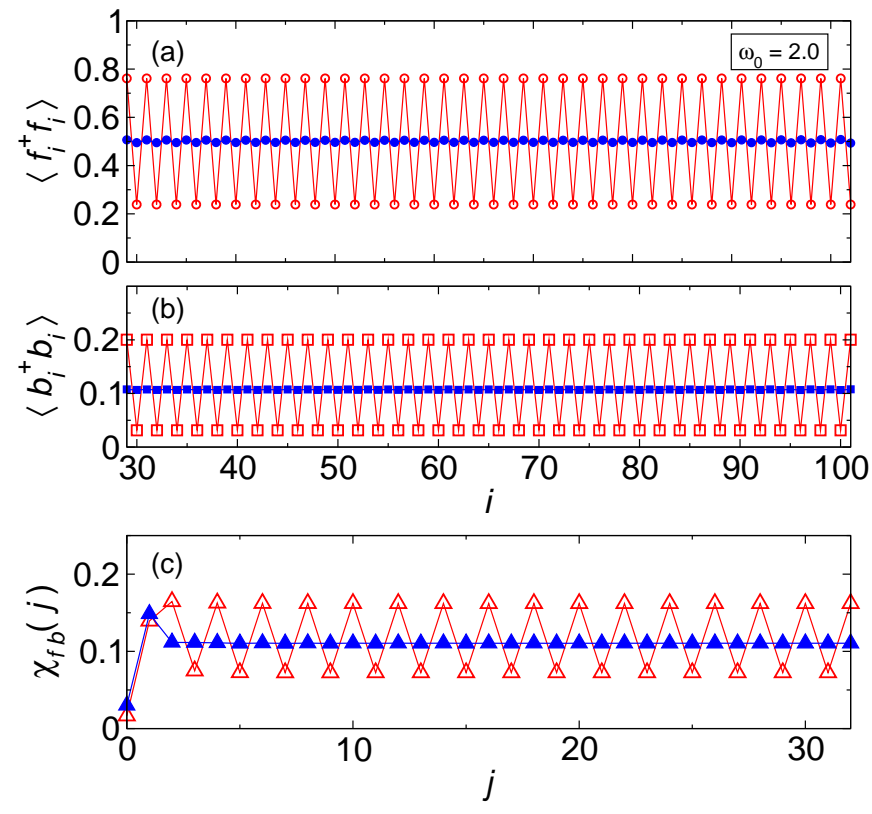

FIG. 1: (color online) Local densities of fermions $\left\langle f_{i}^{\dagger} f_{i}\right\rangle$ (a) and bosons $\left\langle b_{i}^{\dagger} b_{i}\right\rangle$ (b) for a 128-site system with OBC. Open symbols are for $\lambda=0.01$ (CDW regime), filled ones for $\lambda=$ 0.5 (metallic regime). The fermion-boson correlation function $\chi_{f b}(j)$ is given in panel (c) for a 64 -site system with APBC (here the discarded weight is $1.4 \times 10^{-10}\left(7.9 \times 10^{-10}\right)$ for $\lambda=0.01(\lambda=0.50))$. In all cases $\omega_{0}=2.0$.

also in the fermion-boson correlation function

$$
\chi_{f b}(j)=\frac{1}{N_{f}} \sum_{i}\left\langle f_{i}^{\dagger} f_{i} b_{i+j}^{\dagger} b_{i+j}\right\rangle .
$$

Calculating $\chi_{f b}(j)$ at $\omega_{0}=2.0$ for $N=64$ with antiperiodic boundary conditions (APBC) [18], we find a distinctive alternation for $\lambda=0.01$ and a constant value away from the "central site" for $\lambda=0.50$ which again supports the MIT scenario (see Fig. 1 (c)). Note that in the latter case there is still a large boson density at the particle's nearest-neighbor site, locally enhancing the mobility of the carrier.

Whether the pronounced CDW correlations observed for small $\lambda$ and large $\omega_{0}$ are signatures of true long-range order remains an open issue yet. To answer this question, we explore the static charge-structure factor,

$$
S_{c}(q)=\frac{1}{N} \sum_{j, k} e^{i q(j-k)}\left\langle\left(f_{j}^{\dagger} f_{j}-\frac{1}{2}\right)\left(f_{k}^{\dagger} f_{k}-\frac{1}{2}\right)\right\rangle,
$$

where $0 \leq q<2 \pi$. If $S_{c}(\pi) / N$ stays finite in the thermodynamic limit, CDW long-range order exists. Fig. 2 (a) demonstrates that this is the case for $\lambda=0.01$, i.e. when the distortions of the background relax poorly. By contrast, $\lim _{N \rightarrow \infty} S(\pi) / N=0$ for $\lambda=0.5$. This means the model (1) undergoes a quantum phase transition from a metal to an insulator as the relaxation parameter $\lambda$ decreases at fixed $\omega_{0}$. 

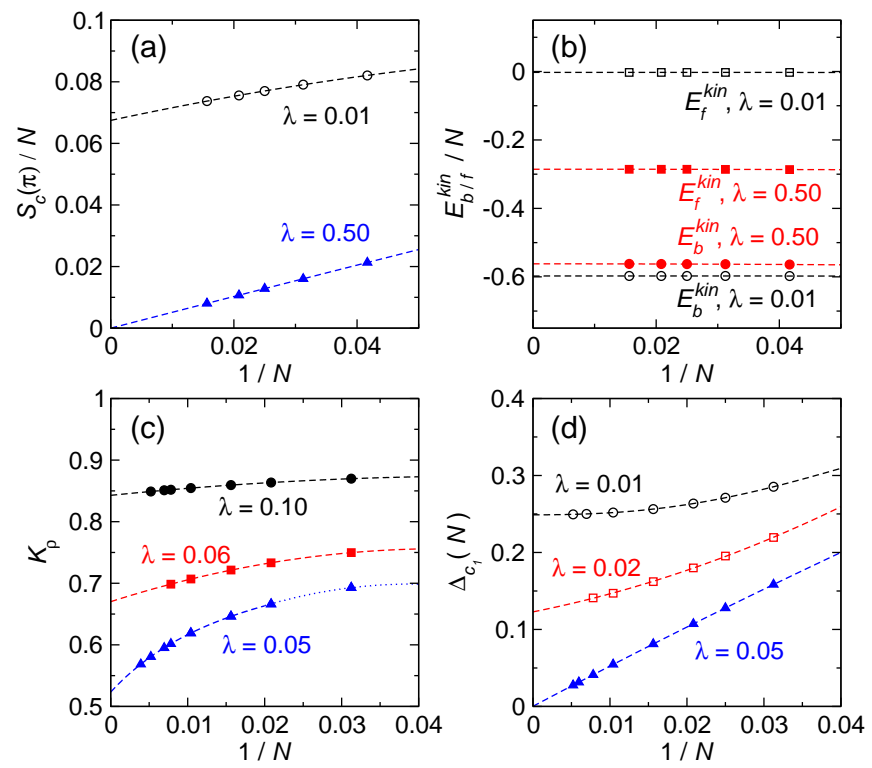

FIG. 2: (color online) Finite-size scaling of several physical quantities: (a) static charge structure factor $S_{c}(q)$ at $q=\pi$, (b) kinetic energy parts $E_{b / f}^{k i n}$, (c) Luttinger liquid parameter $K_{\rho}$, and (d) single-particle excitation gap $\Delta_{c_{1}}$. Data obtained for $\omega_{0}=2$ with APBC $[(\mathrm{a}),(\mathrm{b})]$ and OBC [(c),(d)] applied.

Next we investigate the relative importance of the different transport mechanisms by calculating the kinetic energy parts

$$
E_{b / f}^{k i n}=\left\langle\psi_{0}\left|H_{b / f}\right| \psi_{0}\right\rangle
$$

$\left(\left|\psi_{0}\right\rangle\right.$ denotes the ground state). Fig. 2 (b) shows that $E_{f}^{k i n}$ tends to zero at small $\lambda$, indicating the suppression of the coherent transport channel. Note that bosonassisted transport is possible for both small and large $\lambda$ $\left(E_{b}^{k i n}\right.$ stays close to -0.6 , see panel (b)), and even becomes more pronounced in the CDW phase.

Finally we determine the TLL charge exponent $K_{\rho}$ and the single-particle excitation gap $\Delta_{c_{1}}$ (which, for the model (11), equals the charge gap). $K_{\rho}$ is proportional to the slope of the charge structure factor in the longwavelength limit $q \rightarrow 0^{+}$[19]:

$$
K_{\rho}=\pi \lim _{q \rightarrow 0} \frac{S_{c}(q)}{q}, \quad q=\frac{2 \pi}{N}, \quad N \rightarrow \infty .
$$

From this relation we can calculate $K_{\rho}$ quite accurately using DMRG techniques. As is well-known the 1D spinless fermion model with the nearest-neighbor Coulomb interaction $V$ at half filling can be mapped onto the exactly solvable XXZ model. There the TLL charge exponent decreases from $K_{\rho}=1$, as $V$ is enhanced, and finally reaches $1 / 2$ at the MIT point [20]. We expect that this holds also for the 1D spinless fermion transport model (11) at half band-filling, even though there are only a few analytical or numerical results referring to this for

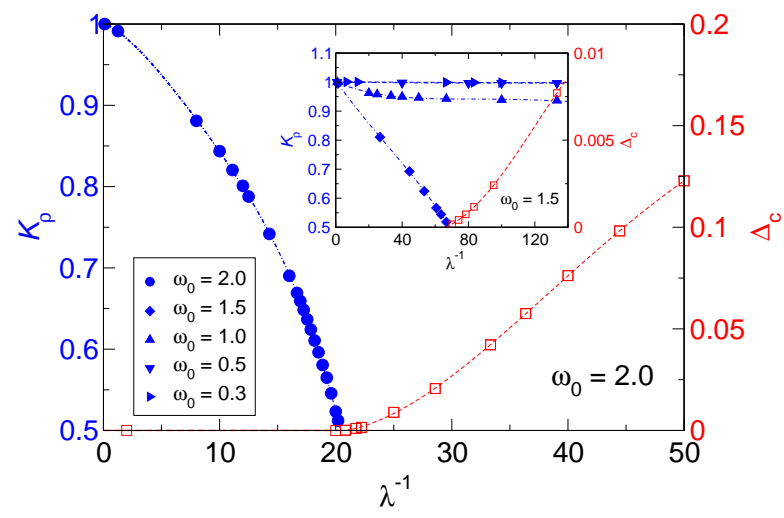

FIG. 3: (color online) Single-particle gap $\Delta_{c_{1}}$ (squares) and TLL parameter $K_{\rho}$ as a function of $\lambda^{-1}$ for $\omega_{0}=2.0$ (main panel). The inset displays results for smaller $\omega_{0}$ and shows that (i) no CDW state is found for $\omega_{0}<\omega_{c}$ and (ii) $K_{\rho}<1$ for all $\omega_{0}$, where $K_{\rho} \rightarrow 1$ as $\omega_{0} \rightarrow 0$ and/or $\lambda^{-1} \rightarrow 0$.

coupled fermion-boson systems (for the (Hubbard-) Holstein model see [6, 10]). The single-particle (charge) gap can be obtained from

$$
\Delta_{c_{1}}(N)=E\left(N_{f}+1\right)+E\left(N_{f}-1\right)-2 E\left(N_{f}\right)
$$

where $E\left(N_{f}\right)$ and $E\left(N_{f} \pm 1\right)$ are the ground-state energies in the $N_{f^{-}}$and $\left(N_{f} \pm 1\right)$-particle sectors, respectively, with $N_{f}=N / 2$. Fig. 2 (c) and (d) illustrate the finitesize scaling analysis for the TLL parameter (c) and the charge gap (d) at $\omega_{0}=2.0$. Both physical quantities can be extrapolated by performing a least-squares fit to a second-order polynomial in $1 / N$. Note that close to the MIT points we need larger system sizes, because a strong finite-size dependence evolves. In this regime, we use chains from $N=32$ to 256 sites and higher order polynomial functions (up to fourth order) to extrapolate the data. In doing so, we determine the non-universal exponents $K_{\rho}(\lambda)>0.5$ in the metallic TLL phase where $\Delta_{c_{1}}=0$, and the finite charge gap $\Delta_{c_{1}}>0$ in the CDW phase (see panels (c) and (d)).

In Fig. 3 we display the extrapolated values of the TLL exponent and the charge gap as a function of $\lambda$ at fixed $\omega_{0}=2.0$. Lowering $\lambda, K_{\rho}$ decreases from $1 \rightarrow 1 / 2$. The point where $K_{\rho}=1 / 2$ is reached marks the critical coupling for the MIT $\left(\lambda_{c}^{-1}\left(\omega_{0}=2\right) \sim 20.4\right)$. From the extrapolated DMRG data it seems that the charge gap opens exponentially on entering the insulating phase and afterwards rises almost linearly. This result is similar to what is observed for the TLL-CDW transition in the anti-adiabatic strong-coupling limit of the spinless fermion Holstein model, which there possesses XXZmodel physics (i.e., a Kosterlitz-Thouless transition at the spin isotropy point). But note that we find a repulsive particle interaction $\left(K_{\rho} \leq 1\right)$ in the metallic phase for small boson frequencies as well, i.e. there is no indication for a pairing instability in the half-filled band case. 


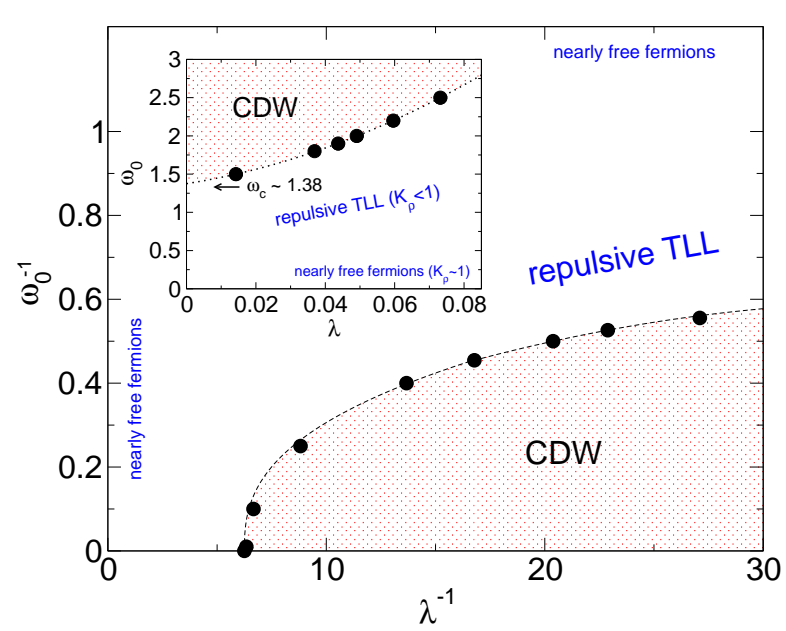

FIG. 4: (color online) DMRG phase diagram of the twochannel transport model (1) for the 1D half-filled band case (the dashed line is a guide to the eye). The inset gives the phase diagram in the $\lambda-\omega_{0}$ plane. The MIT point for $\lambda=0$, $\omega_{0}(0) \sim 1.38$, is obtained from a quadratic fit (dotted line).

Figure 4 represents the main result of our work: The ground-state phase diagram of the 1D half-filled fermionboson model (1) in the $\lambda^{-1}-\omega_{0}^{-1}$ plane. Obviously the phase space is divided into two regimes, the metallic TLL phase and the insulating CDW phase with long-range order. We first discuss the limit of large $\omega_{0}$. In this regime background fluctuations, which are intimately connected with any particle hop, are energetically costly. As a result the itinerancy of the particles is suppressed to a large extent and charge ordering becomes favorable. Nevertheless, we find a metallic state even for $\omega_{0}=\infty$ provided that $\lambda^{-1}<\lambda_{c}^{-1}\left(\omega_{0}=\infty\right.$ ) (numerically we proved the TLL to exist for $\lambda^{-1}<\lambda_{c}^{-1} \simeq 6.3$ at $\left.\omega_{0}=1000\right)$. In this case the system's ability for relaxation $(\propto \lambda)$ is strong enough to prevent long-range charge order. This is reminiscent of the existence of a finite critical coupling strength $g_{c}\left(g^{2}=\varepsilon_{p} / \omega_{0}\right)$ in the antiadibatic limit $\left(\omega_{0} \rightarrow \infty\right)$ of the spinless fermion Holstein model, where the TLL phase is realized for $g<g_{c}(\omega)$ [6]. In contrast to the TLL-CDW transition in the Holstein model, however, the symmetry-broken CDW state is a few-boson state 14 (i.e., not a Peierls phase with many phonons involved). In the opposite limit of small $\omega_{0}$, the background medium is easily disturbed by particle motion. Therefore the rate of bosonic fluctuations $\left(\propto \omega_{0}^{-1}\right)$ is high. Now we enter the fluctuation dominated regime [11], and consequently CDW order is suppressed. The inset of Fig. [4 shows that even for $\lambda=0$, i.e. if the explicit $\lambda$-relaxation channel is closed, a metallic state may exist below a finite critical energy $\omega_{0}(0)$.

To conclude, using an unbiased numerical (DMRG) technique, we proved that the very general fermion-boson transport model (11) displays a correlation-induced metal insulator transition at half filling in 1D. The metallic phase typifies a repulsive Luttinger liquid, while the insulating phase shows CDW long-range order. The phase boundary between these states is non-trivial. It would be highly desirable to verify the numerical results of this paper by an analytical (field theoretical or algebraic) approach.

Acknowledgments. The authors would like to thank A. Alvermann, D. M. Edwards, E. Jeckelmann, S. Nishimoto, and G. Wellein for valuable discussions. This work was supported by DFG through SFB 652, and the KONWIHR project HQS@HPC. S. E. acknowledges funding by Ministerium für Bildung, Wissenschaft und Kultur Mecklenburg-Vorpommern, Grant No. 0770/461.01.

[1] F. Bloch, Z. Phys. 57, 545 (1929); A. H. Wilson, Proc. Roy. Soc. London, Ser. A 133, 458 (1931).

[2] P. W. Anderson, Phys. Rev. 109, 1492 (1958).

[3] N. F. Mott, Metal-Insulator Transitions (Taylor \& Francis, London, 1990); F. Gebhard, The Mott MetalInsulator Transition, vol. 137 of Springer tracts in modern physics (Springer, 1997).

[4] R. Peierls, Quantum theory of solids (Oxford University Press, Oxford, 1955).

[5] J. Fröhlich, F. Martinelli, E. Scoppola, and T. Spencer, Commun. Math. Phys. 101, 21 (1985).

[6] R. J. Bursill, R. H. McKenzie, and C. J. Hamer, Phys. Rev. Lett. 80, 5607 (1998); M. Hohenadler, G. Wellein, A. R. Bishop, A. Alvermann, and H. Fehske, Phys. Rev. B 73, 245120 (2006).

[7] F. H. L. Essler, H. Frahm, F. Göhmann, A. Klümper, and V. E. Korepin, The One-Dimensional Hubbard Model, Cambridge University Press, Cambridge, 2005).

[8] F. Gebhard and A. E. Ruckenstein, Phys. Rev. Lett. 68, 244 (1992).

[9] S. Ejima and S. Nishimoto, Phys. Rev. Lett. 99, 216403 (2007).

[10] M. Tezuka, R. Arita, and H. Aoki, Phys. Rev. Lett. 95, 226401 (2005); R. T. Clay and R. P. Hardikar, Phys. Rev. Lett. 95, 096401 (2005); H. Fehske, G. Hager, and E. Jeckelmann, Europhys. Lett. 84, 57001 (2008).

[11] D. M. Edwards, Physica B 378-380, 133 (2006); A. Alvermann, D. M. Edwards, and H. Fehske, Phys. Rev. Lett. 98, 056602 (2007).

[12] S. A. Trugman, Phys. Rev. B 37, 1597 (1988).

[13] G. Martinez and P. Horsch, Phys. Rev. B 44, 317 (1991).

[14] G. Wellein, H. Fehske, A. Alvermann, and D. M. Edwards, Phys. Rev. Lett. 101, 136402 (2008).

[15] S. R. White, Phys. Rev. Lett. 69, 2863 (1992).

[16] E. Jeckelmann and S. R. White, Phys. Rev. B 57, 6376 (1998); E. Jeckelmann and H. Fehske, Rivista del Nuovo Cimento 30, 259 (2007).

[17] The use of smooth boundary conditions opens another possibility to reduce oscillatory finite-size effects, see M. Vekić and S. R. White, Phys. Rev. Lett. 26, 4283 (1993).

[18] Note that APBC give the lowest energy for our spinless fermion model with $N_{f}=4 n$ particles and $2 N_{f}$ sites.

[19] M. Dzierzawa, in The Hubbard model, edited by D. Baeriswyl et al. (Plenum, New York, 1995), vol. 343 of NATO Advanced Study Institutes, Ser. B.

[20] S. Ejima, F. Gebhard, and S. Nishimoto, Europhys. Lett. 
70, 492 (2005). 